\title{
METODOLOGÍA MIXTA FLIPPED CLASSROOM Y APRENDIZAJE BASADO EN PROYECTOS PARA EL APRENDIZAJE DE LA GEOMETRÍA ANALÍTICA EN SECUNDARIA
}

\author{
Mixed Flipped Classroom and Project-Based Learning \\ Methodology for Learning Analytical Geometry \\ in Secondary Schools
}

\author{
Álvaro ANTón SANCHO* y Marina SÁnCHEZ DOMíNGUEZ*** \\ * Dpto. Matemáticas y Ciencia Experimental. Escuela Universitaria de Magisterio \\ "Fray Luis de León". Universidad Católica de Ávila \\ *alvaro.anton@ucavila.es \\ *marinaparab@gmail.com
}

Recibido: 05/12/2020; Aceptado: 17/12/2020; Publicado: 27/12/2020

Ref. Bibl. ÁlVARO ANTÓN SANCHO y MARINA SÁNCHEZ DOMÍNGUEZ. Metodología mixta Flipped Classroom y aprendizaje basado en proyectos para el aprendizaje de la geometría analítica en secundaria. Enseñanza ETeaching, 38, 2-2020, 135-156.

RESUMEN: Este trabajo se enmarca en el contexto de los proyectos de innovación educativa dentro de la didáctica específica de las matemáticas en el nivel de Educación Secundaria Obligatoria. En concreto, en este artículo proponemos, y llevamos a la práctica en una experiencia de aula, las líneas metodológicas básicas de lo que se podría denominar una metodología mixta que se basa en los principios y fases del Aprendizaje Basado en Proyectos, pero introduciendo en el proceso, como un elemento intrínsecamente ligado a él, acciones didácticas realizadas según el método de Flipped Classroom. La primera parte del trabajo analiza las dos perspectivas metodológicas citadas y describe las etapas que proponemos seguir para la implementación de la metodología mixta. La parte principal del artículo, por su 
ÁLVARO ANTÓN SANCHO Y MARINA SÁNCHEZ DOMÍNGUEZ

METODOLOGÍA MIXTA FLIPPED CLASSROOM Y APRENDIZAJE BASADO EN PROYECTOS

PARA EL APRENDIZAJE DE LA GEOMETRÍA ANALÍTICA EN SECUNDARIA

parte, se ocupa de describir una intervención educativa en la que se implementó una metodología como la descrita, combinada de Aprendizaje Basado en Proyectos con el modelo de Flipped Classroom, en un conjunto de alumnos de $4 .^{\circ}$ de ESO para trabajar contenidos curriculares de geometría analítica, dentro del área de matemáticas. En la intervención se han empleado instrumentos de pretest-postest para valorar la eficacia de la metodología empleada. Los resultados obtenidos evidencian que se ha logrado un incremento significativo de los conocimientos de los alumnos en el área tratada derivado de la intervención. Los resultados de este artículo están basados en los contenidos presentados por la segunda autora en su Trabajo Fin de Máster de Profesorado de Educación Secundaria en la Universidad Católica de Ávila en junio de 2020 (Sánchez, 2020).

Palabras clave: Aprendizaje Basado en Proyectos; Flipped Classroom; geometría; educación secundaria; innovación educativa.

SUMMARY: This work is part of the educational innovation projects within the specific didactics of mathematics at the Compulsory Secondary Education level. Specifically, in this paper we propose, and put into practice in a classroom experience, the basic methodological lines of what could be called a mixed methodology based on the principles and phases of Project-Based Learning, but introducing into the process, as an element intrinsically linked to it, didactic actions carried out according to the Flipped Classroom method. The first part of the paper analyses the two methodological perspectives mentioned above and describes the stages we propose to follow for the implementation of the mixed methodology. The main part of the paper, for its part, deals with describing an educational intervention in which a methodology such as the one described, combined Project-Based Learning with the Flipped Classroom model, was implemented in a group of 4th year ESO students to work on curricular contents of analytical geometry, within the area of mathematics. In the intervention, pretest-postest instruments have been used to evaluate the effectiveness of the methodology employed. The results obtained show that a significant increase in the students' knowledge in the area treated has been achieved as a result of the intervention. The results contained in this paper are based in the contents presented by the second author in her Final Project for the Master's Degree in Secondary Education Teaching at the Catholic University of Ávila in June 2020 (Sánchez, 2020).

Key words: Project-Based Learning; Flipped Classroom; Geometry; Secondary Education Level; Educational Innovation.

\section{INTRODUCCIÓN}

Los sucesivos informes PISA muestran que las destrezas matemáticas y científicas del alumnado español de enseñanzas medias se encuentran estancadas en niveles siempre por debajo de la media de los países de nuestro entorno, pese a las reformas curriculares que la asignatura de matemáticas ha sufrido en España en los últimos veinte años. El último informe de la OCDE (2020) pone el acento 
en las características actitudinales que los alumnos españoles presentan ante los contenidos matemáticos como razón principal de este estancamiento, siendo así que este hecho no se debe tanto a aspectos epistemológicos, derivados de la dificultad propia de la materia o vinculados a la comprensión lectora, variables todas ellas que demuestran ser poco significativas para explicar los mediocres resultados al respecto. Esta observación es coherente con las ideas de algunos estudios recientes, como Gómez-Chacón (2000) o Morales y García (2013), que ponen de manifiesto la existencia de un ć́rculo vicioso de rendimiento bajo en matemáticas que redunda en un autoconcepto negativo de las propias capacidades del alumno hacia ellas, todo ello iniciado en un contexto de falta de motivación inicial.

La literatura académica específica sobre educación matemática viene indicando la necesidad de realizar reformas metodológicas en la enseñanza de las matemáticas, encaminadas, principalmente, a la atribución progresiva de un rol cada vez más activo del alumno (Vaello, 2009). En este sentido, resulta especialmente interesante el método del Aprendizaje Basado en Proyectos (en adelante, $\mathrm{ABP}$ ), cuya perspectiva se fundamenta en desarrollar los contenidos curriculares a través de proyectos llevados a cabo por los alumnos (Mettas y Constantinou, 2007). En los últimos años ha aumentado también la aplicación de un modelo metodológico llamado Flipped Classrooom (en adelante, FC), basado en suscitar del alumno que trabaje los contenidos de manera autónoma a partir de materiales proporcionados por el profesor, con buenos resultados de aprendizaje en diferentes áreas de conocimiento (Aguilera-Ruiz et al., 2017). Nuestro propósito en este trabajo es exponer una experiencia de aula en la que se ha utilizado una metodología mixta, fundamentada en ABP, pero introduciendo una dinámica de FC en el aula para la enseñanza de contenidos de geometría analítica en un alumnado de $4 .^{\circ}$ de ESO. Hemos hecho un estudio pretest-postest seguido de un contraste de hipótesis para verificar si la implementación de un modelo como este permite un incremento significativo del aprendizaje de los citados contenidos en el alumnado.

Para ello, hemos estructurado el artículo del siguiente modo: en las secciones 2, 3 y 4 se presentan, respectivamente, las líneas fundamentales de las metodologías del ABP, del FC y de la metodología mixta que pretendemos introducir y aplicar. Finalmente, la sección 5 se ocupa de describir la intervención educativa que hemos llevado a cabo en $4 .^{\circ}$ de ESO y de analizar los resultados que hemos obtenido.

\section{FLIPPED CLASSROOM}

La metodología FC, aplicada a los diferentes niveles educativos, es relativamente reciente: la denominación, que suele traducirse como «clase inversa» o «clase al revés", fue originalmente establecida por Lage, Platt y Treglia (2000), aunque los primeros estudios que afianzaron su uso datan del año 2007 (Hamdan, McKnight, McKnight y Arfstrom, 2013). La idea fundamental consiste en desarrollar materiales de trabajo a través de los cuales el alumno sea capaz de desarrollar su propio 
ÁLVARO ANTÓN SANCHO Y MARINA SÁNCHEZ DOMÍNGUEZ

METODOLOGÍA MIXTA FLIPPED CLASSROOM Y APRENDIZAJE BASADO EN PROYECTOS

PARA EL APRENDIZAJE DE LA GEOMETRÍA ANALÍTICA EN SECUNDARIA

estudio acerca del tema que se trate para compartir posteriormente los conceptos aprendidos y sus dudas al respecto en el contexto del grupo-clase, afianzando, de este modo, sus conocimientos. Se favorece, así, como principio esencial del modelo, la participación activa del alumnado.

Siguiendo la dinámica del modelo de aula inversa que hacen Santiago y Tourón (2015) y, más extensa y detalladamente, Martín y Calvillo (2017), podemos sintetizar, como las grandes fases que vertebran la metodología FC, las siguientes: selección de contenidos a trabajar por los alumnos y diseño de materiales (presentaciones, textos, vídeos, etc.) para reforzar su comprensión; preparación de explicaciones adecuadas a los alumnos (donde es útil el uso de ejemplos prácticos o vídeos adicionales); trabajo autónomo del alumno a partir de los materiales proporcionados, y planificación de una clase presencial mediante acciones prácticas que permitan el afianzamiento de lo aprendido.

Rigo, Riccetti, Siracursa y Paoloni (2019), citando los estudios realizados por Cronhjort, Filipsson y Weurlander (2018), García (2016), Hutchings y Quinney (2015) y James, Chin y Willliams (2014), concluyen que una metodología tipo FC promueve la implicación de los estudiantes con la materia de conocimiento e incrementa las oportunidades de su afianzamiento, porque favorece la introducción de tiempos para la discusión y la interacción con el profesor, aspecto que ya habían puesto de relieve otros autores y estudios recientes como Flummerfelt y Green (2013), Leicht et al. (2012), Phillips y Trainor (2014) o Roehl, Reddy y Shannon (2013). Por su parte, Wilson (2013) manifiesta que esta metodología conlleva una mejora en el rendimiento general del alumnado, medido en términos de las calificaciones de las asignaturas de su plan formativo. En lo que respecta al aprendizaje específico de las matemáticas en la educación secundaria, hay trabajos recientes, como el ya citado de Wilson (2013), o como el de Fornons y Palau (2016), que demuestra, a partir de un estudio práctico cuantitativo llevado a cabo en un grupo de $3 .^{\circ}$ de ESO, que la metodología FC suscita un incremento significativo en las calificaciones de la asignatura de matemáticas (en el caso concreto de este estudio, un $20,7 \%$ respecto de un grupo control en el que se desarrolló la metodología tradicional).

\section{APRENDIZAJE BASADO EN PROYECTOS}

Por su parte, la metodología ABP se fundamenta en el hecho de que los alumnos tienen que trabajar de forma cooperativa y colaborativa sobre una situación didáctica, a modo de proyecto o problema inicialmente planteado, aunque la definición definitiva del problema o proyecto sea fruto de las ideas que, al respecto, sean propuestas por el propio alumnado, y de forma que el contexto en que se sitúe sea lo más realista y significativo posible. De inspiración constructivista, el modelo pone el acento, además, en una adecuada organización del ambiente y de los alumnos, habitualmente divididos en grupos de trabajo, equilibrados en cuanto a las capacidades, talentos y resultados académicos de cada uno, y de forma que 
haya una atribución de roles, frecuentemente decidida por el profesor, aunque no necesariamente. Normalmente se sugiere, además, la supervisión del proceso mediante la elaboración de un diario de registro de la actividad realizada (Dole, Bloom y Kowalske, 2016).

Thomas (2000) explica que las metodologías tradicionales han incorporado desde siempre actividades encaminadas a favorecer el aprendizaje de los alumnos mediante acciones colaborativas de investigación, pero que estas siempre han sido excepcionales y subordinadas al desarrollo de los contenidos curriculares, como ejercicios de aplicación de los mismos. En este sentido, Thomas (2000) desarrolla cinco aspectos de la metodología del ABP que señala como definitorios y distintivos respecto de estas otras actividades propias de las metodologías tradicionales:

- Los proyectos centran metodológicamente el desarrollo del currículo, frente a la subordinación tradicional.

- El fundamento de los proyectos son las preguntas orientadas, que dirigen a los estudiantes, sin coartar su libertad de actuación, en el proceso de elaboración de sus propios conocimientos al respecto de los contenidos que se pretende trabajar.

- Esa elaboración de conocimientos es constructiva y no pasiva.

- En los proyectos, incluso en su propia definición, es crucial el impulso que introducen los alumnos.

- Los proyectos deben tender a ser realistas y significativos.

Nuestra pretensión es introducir estas claves metodológicas como vertebradoras de nuestra metodología mixta basada en proyectos y clase inversa. Asimismo, incorporaremos las principales fases que, en el desarrollo de los proyectos, conviene plantear, a saber: presentación y planteamiento del problema y posterior debate que conduzca a una lluvia de ideas para la definición definitiva del mismo y la clarificación de dudas; identificación de las situaciones problemáticas planteadas y de los recursos necesarios para su resolución; desarrollo del plan de investigación según los roles asignados; presentación de los resultados. Mantendremos, de igual modo, el diario reflexivo como principal instrumento de seguimiento.

Son muy numerosas las investigaciones que, desde finales de los años 90, se han realizado en orden a demostrar la efectividad del ABP en las diferentes etapas educativas y áreas de conocimiento, muchas de ellas basadas en experiencias de aula (Thomas, 2000). Por cercanía temática, citaremos, de entre ellas, el trabajo de Barron et al. (1998), que reporta la experiencia llevada a cabo durante cinco semanas en la que los alumnos trabajaron en un proyecto sobre conceptos geométricos presentados en el contexto de problemas arquitectónicos. El estudio revela un incremento cuantitativamente significativo en los estudiantes (medido a partir de pretest y postest) de las capacidades de medición, la comprensión de escalas y el cálculo de perímetros, áreas, volúmenes y otras magnitudes geométricamente significativas. Por su parte, Boaler $(1998,2002)$ estudió el rendimiento en matemáticas en los estudiantes británicos de entre 13 y 16 años de una escuela secundaria que 
trabajaba con ABP y lo comparó con los correspondientes datos de rendimiento de otra escuela de la misma etapa que trabajaba con la metodología tradicional. Sus resultados muestran que, partiendo de niveles semejantes en matemáticas (evaluados mediante una prueba estandarizada nacional de habilidades matemáticas), los alumnos de la escuela que trabajaba por proyectos alcanzaron unas puntuaciones mejores en matemáticas a lo largo de los tres años del estudio y demostraron mejores habilidades vinculadas a procesos que requieren el uso práctico de conceptos matemáticos y, sorprendentemente, también mejor capacidad de memorización de conceptos matemáticos. Otros estudios más recientes han permitido concluir que el $\mathrm{ABP}$ redunda también en una mejor percepción del área de matemáticas por parte de los alumnos y, consecuentemente, una mayor motivación hacia su estudio. Es el caso de Morales y García (2015), que reportan datos a este respecto evaluados en alumnos panameños de enseñanza media a través de un proyecto de estudio de aspectos trigonométricos en un contexto arquitectónico. Otros estudios demuestran que la introducción de herramientas TIC en la presentación de los proyectos de trabajo o como recursos otorgados a los alumnos para el desarrollo de su investigación resulta algo natural en el ABP e incrementa su eficacia como metodología en la enseñanza de las matemáticas (Badia y García, 2006).

\section{Metodología miXta Flipped Classroom y Aprendizaje Basado en Proyectos}

\subsection{Presentación de la metodología mixta}

La metodología mixta FC y ABP que vamos a desarrollar parte de la base de los principios básicos del ABP que ya hemos explicado (esencialmente, trabajo autónomo y cooperativo en grupos basado en proyectos o problemas significativos que requieren de un proceso de investigación por parte del alumno y de modo que se cubran los contenidos curriculares de nuestro interés), pero de forma que algunas de las acciones que se desarrollan (en concreto, el momento inicial de motivación y la preparación y exposición de los contenidos teóricos necesarios) se lleven a cabo según el modelo de FC. Buscamos, de esta manera, integrar los beneficios que han demostrado reportar ambas metodologías para el aprendizaje de las matemáticas en la educación secundaria.

En concreto, la metodología que queremos desarrollar tendría una fase inicial de introducción a los contenidos que se pretendan trabajar, mediante un vídeo, explicación o documentos de trabajo proporcionados por el profesor, seguido de un debate y lluvia de ideas al respecto. Posteriormente, los alumnos trabajarán de manera cooperativa en pequeños grupos, cada uno a propósito del proyecto que le haya tocado en una previa distribución de los mismos. Parte de ese proyecto consiste en preparar material para proceder después a explicar ante el resto de los alumnos ciertos contenidos vinculados al proyecto, de modo que las sesiones combinarán tiempo dedicado al trabajo autónomo en los proyectos y tiempo dedicado a la exposición de materiales y contenidos por parte de los alumnos. 
De la forma que acabamos de describir, la vinculación entre FC y ABP no es de mera adición o complementariedad, sino que se establece una interconexión esencial entre ambas metodologías. No se trata, por tanto, de aplicar conjuntamente los dos métodos, sino de diseñar una nueva perspectiva metodológica que integre los principios fundamentales de ambos. Hay varios aspectos que muestran esta esencial vinculación. Por ejemplo, el hecho de que la responsabilidad sobre la adquisición de conocimientos de los alumnos recae, de modo importante, sobre los propios alumnos, ya que son ellos mismos los que diseñan la materia de aprendizaje para el resto, se encargan de explicar los contenidos y deben asegurarse de que el resto de los alumnos los entienden. Además, los alumnos deben someterse a las preguntas de sus compañeros, siendo así que las consideraciones que estos hagan pueden influir en la definición del producto final del proyecto del grupo correspondiente. Queda a criterio del profesor que el grupo lleve a cabo una exposición final de su proyecto. Asimismo, el aprendizaje que los alumnos lleven a cabo de los contenidos explicados por otros grupos puede influir en el desarrollo y resultado final de su propio proyecto. Dicho de otro modo, una transmisión errónea o deficiente de contenidos repercutirá negativamente en los resultados finales de los proyectos, así como en los resultados de aprendizaje.

\subsection{Etapas de la metodología mixta FC y ABP}

Tomando las estructuras típicas de los proyectos como base (Thomas, 2000) y las ideas fundamentales del método FC que hemos desarrollado ya, podemos establecer, a nivel general, las siguientes como etapas de una acción educativa basada en la metodología mixta que acabamos de presentar:

\subsubsection{Etapa 1. Presentación de los contenidos básicos}

Se trata de realizar una presentación de los contenidos que pretenden trabajarse a lo largo de la acción educativa, de un modo suficientemente abierto para que, por un lado, suscite la curiosidad de los alumnos, les plantee cuestiones problemáticas sobre las que investigar y les motive a buscar recursos para su resolución.

En esta etapa, el profesor puede basarse en vídeos tutoriales, en explicaciones apoyadas en presentaciones tipo Prezi o realizadas mediante el método tradicional, e incluso puede combinar estos recursos.

\subsubsection{Etapa 2. Lluvia de preguntas y debate inicial}

Inmediatamente después de la actividad de introducción del contenido, se abrirá un tiempo de debate y lluvia de ideas, apoyado en una o varias preguntas suscitadas por el profesor. De esta sinergia comunicativa entre el profesor y los 

PARA EL APRENDIZAJE DE LA GEOMETRÍA ANALÍTICA EN SECUNDARIA

alumnos debe surgir la definición precisa de los proyectos sobre los que posteriormente se deberá trabajar.

\subsubsection{Etapa 3. Organización grupal y presentación del proyecto a los grupos}

El grupo-clase debe ser organizado, preferentemente, en pequeños grupos, siguiendo, para la distribución de estos, el criterio establecido por el profesor, que, en todo caso, debe tener en cuenta el rol social de cada alumno en la clase, de modo que haya diversidad de roles en cada grupo. Esto ayudará a que el resultado final del trabajo sea más eficiente y a que, en el proceso, se lleve a cabo una adquisición de conocimientos lo más homogénea posible. Asimismo, se propondrán los proyectos que los grupos deberán realizar y los contenidos que se deberán preparar para la exposición a sus compañeros.

Para la distribución concreta de los alumnos en los grupos, es deseable que se formen, usando la terminología propia de la estructuración de grupos cooperativos (Pujolàs, 2004), equipos base o equipos de expertos. Los equipos base son grupos de unos tres o cuatro miembros, heterogéneos en cuanto al rol social de cada uno y permanentes. Básicamente, deberíamos hacer una distribución ficticia de los alumnos en tres grandes bloques, ilustrados en la Tabla 1, y formar los grupos escogiendo uno o dos alumnos de la columna central y un alumno de cada una de las columnas de los extremos.

TABLA 1

Formación de equipos base

\begin{tabular}{c|c|c}
\hline Bloque 1 & Bloque 2 & Bloque 3 \\
\hline $\begin{array}{c}\text { Alumnos con iniciativa, } \\
\text { capacidad de liderazgo, } \\
\text { motivación y entusiasmo }\end{array}$ & $\begin{array}{c}\text { Resto de los } \\
\text { alumnos }\end{array}$ & $\begin{array}{c}\text { Alumnos pocos motivados y con bajo } \\
\text { rendimiento escolar o con alguna } \\
\text { necesidad específica de aprendizaje }\end{array}$ \\
\hline
\end{tabular}

Como variante de la distribución de equipos base, pueden establecerse grupos de expertos. Esto exige que en cada grupo haya un alumno que sea «experto» en el área de contenidos que se vaya a trabajar, en el sentido de que haya demostrado tener una especial facilidad para su aprendizaje o un nivel de conocimientos al respecto significativamente superior al resto de miembros del grupo.

4.2.4. Etapa 4. Lluvia de preguntas y detección de las situaciones problemáticas y de lo que se necesita saber

Una vez se propongan los proyectos a trabajar, se realizará una nueva lluvia de preguntas para que los alumnos puedan solventar todas las cuestiones dudosas que 

PARA EL APRENDIZAJE DE LA GEOMETRÍA ANALÍTICA EN SECUNDARIA

surjan referentes a la definición del proyecto. Esta lluvia de preguntas está pensada para favorecer la detección de las situaciones problemáticas que puedan surgir.

\subsubsection{Etapa 5. Plan de investigación}

Una vez definidos los grupos de trabajo y realizado el reparto de proyectos y de contenidos a exponer, los alumnos deberán distribuir los roles internos dentro del grupo de cara a la realización del proyecto y buscar las herramientas adecuadas para su desarrollo, así como llevar a cabo las correspondientes tareas de investigación, recopilación de información y estructuración del producto final, del mismo modo en que se llevaría a cabo un proyecto típico.

\subsubsection{Etapa 6. Exposición de los contenidos por parte de los alumnos}

El principal objetivo de esta etapa es la transmisión de contenidos por cada grupo de la clase al resto de sus compañeros. Las diferentes exposiciones orales aportan contenidos al resto de compañeros que pueden ser útiles para el desarrollo de sus propios proyectos. A su vez, los comentarios y preguntas de los compañeros pueden servir para mejorar el proyecto del grupo que está haciendo la exposición oral. Esta etapa favorece la detección de situaciones problemáticas o desarrollos erróneos para que el profesor o los miembros del propio grupo de alumnos que esté explicando el contenido en ese momento los identifiquen y puedan disponer los medios adecuados para su resolución o corrección.

\subsubsection{Etapa 7. Desarrollo del producto final}

La finalidad principal de esta etapa es la elaboración definitiva del producto final del proyecto. En este nivel de desarrollo se supone que los alumnos ya tienen las herramientas y el conocimiento necesarios para finalizarlo.

\subsubsection{Etapa 8. Diario reflexivo}

A lo largo de todo el proceso, los grupos de alumnos deberán realizar un diario reflexivo donde registrarán las tareas que han realizado diariamente, los roles que cada uno ha desempeñado, las dificultades que han encontrado y cómo las han superado, y recopilarán todos los datos que estimen necesarios para una completa descripción del proceso. 
ÁLVARO ANTÓN SANCHO Y MARINA SÁNCHEZ DOMÍNGUEZ

METODOLOGÍA MIXTA FLIPPED CLASSROOM Y APRENDIZAJE BASADO EN PROYECTOS PARA EL APRENDIZAJE DE LA GEOMETRÍA ANALÍTICA EN SECUNDARIA

\subsubsection{Etapa 9. Evaluación del aprendizaje}

Se trata de evaluar la adquisición de conocimientos y competencias por parte de cada alumno al respecto de los contenidos que se hayan introducido a través de la acción educativa. Esta evaluación tendrá en cuenta el seguimiento realizado a través de la observación directa (particularmente, las exposiciones de los diferentes grupos), el diario reflexivo y una prueba objetiva final de contenidos, que puede llevarse a cabo de manera oral o escrita, en papel o a través de alguna herramienta digital conocida por los alumnos.

\subsection{Uso de herramientas digitales}

A lo largo de todo el proceso, conviene integrar herramientas de tecnología educativa que asistan en la correcta realización de cada una de las etapas. A continuación, presentamos, a modo de ejemplo, una recopilación de este tipo de herramientas TIC:

- Gestión de contenidos. En este tipo de estrategias metodológicas, es imprescindible el uso de herramientas para difundir y gestionar el contenido necesario para que se lleve a cabo el producto final y para que el proceso de aprendizaje sea eficiente. Estas herramientas pueden ser en formato papel, como libros y cuadernos, o en formato digital, como blogs y redes sociales, o como plataformas de uso específicamente educativo, tipo Classroom, Moodle o Edmodo.

- Edición de contenidos. Este tipo de herramientas resultan fundamentales para diseñar, modificar, actualizar y ampliar los contenidos que se pretende que los alumnos desarrollen o expongan al resto de la clase. Entre ellas, las principales son PowerPoint, Prezi, Genially o el mural tradicional.

- Bancos de contenidos. El propósito de estas herramientas es almacenar información y materiales necesarios, tanto para el aprendizaje de los diferentes contenidos como para la creación y elaboración del proyecto a realizar. Algunas de estas herramientas son Classroom, Moodle, Symbaloo, Drive, Wiki o ClassDojo.

- Instrumentos de evaluación. Además de los tradicionales instrumentos, en formato papel u oral, pueden emplearse herramientas digitales más adecuadas al entorno vivencial del alumno y al tipo de recursos que ha empleado durante el proceso de desarrollo de la acción educativa. Algunas de ellas son Kaboot, formularios de Google o Plickers.

- Comunicación. Es conveniente disponer de un canal de referencia a través del cual los alumnos puedan plantear dudas fuera del horario lectivo, en aras de favorecer la fluidez comunicativa entre el profesor y el alumnado y minimizar la ratio de intervención. Además del correo electrónico, se pueden establecer foros en plataformas que los permitan, como Moodle. 


\section{INTERVENCIÓN EDUCATIVA}

\subsection{Participantes y contexto}

La intervención educativa que se va a describir se ha desarrollado en un centro concertado y laico de Educación Infantil, Primaria y Secundaria situado en la provincia de Sevilla.

Se ha llevado a cabo la intervención en dos grupos de $4 .^{\circ}$ de ESO formados por 30 alumnos cada uno, de entre 15 y 16 años, cuya situación económica y social es media-elevada. La muestra que vamos a considerar está formada por 50 de entre esos alumnos (el resto se han extraído del estudio por haberse ausentado a parte o la totalidad de las actividades formativas de la intervención). Se ha escogido este nivel porque se corresponde con un grado madurativo de los estudiantes suficiente como para valorar los efectos de la metodología de nuestro interés y porque nos va a permitir evaluar su eficacia en un alumnado diverso en cuanto al itinerario académico que han escogido $y$, por tanto, en lo que se refiere a su interés por las matemáticas y sus campos afines. Una de las clases consta principalmente de alumnos cuyo itinerario formativo está ligado a las ciencias y el otro grupo, más heterogéneo desde el punto de vista del itinerario académico elegido, lo constituyen alumnos con diferentes proyecciones educativas y profesionales, como la formación profesional o estudios medios o superiores de la rama de ciencias o de ciencias sociales.

De entre los alumnos que forman la muestra, únicamente dos presentan necesidades especiales de aprendizaje: un alumno con TDAH y un alumno que presenta un retraso madurativo. Ambos han participado con normalidad de la intervención, sin que haya sido necesario realizar adaptación curricular alguna ni actividades de refuerzo. Únicamente se realizó una disminución de contenidos en la evaluación (posterior al postest). Además, algunos alumnos son repetidores. Con ellos únicamente ha sido necesario realizar seguimiento diario y personalizado de su rendimiento académico.

Los alumnos de cada una de las dos clases fueron distribuidos en ocho grupos de entre tres y cuatro alumnos por grupo, siguiendo la perspectiva de la sección 4.2 anterior.

\subsection{Diseño}

\subsubsection{Objetivos, variables e hipótesis}

En consonancia con lo dispuesto en el artículo 11 del Real Decreto 1105/2014, de 26 de diciembre, por el que se establece el currículo básico de la Educación Secundaria Obligatoria y del Bachillerato, y siguiendo a Sánchez (2020), buscamos los siguientes objetivos didácticos generales para nuestra intervención: 
- Utilizar adecuadamente la terminología específica del álgebra y la geometría vectoriales.

- Comprender la noción topológica de dimensión a través de objetos vectoriales.

- Reconocer los elementos notables y los conceptos de dependencia lineal y de equipolencia referidos a vectores del plano y en el espacio.

- Comprender y manejar el álgebra propia de los vectores del plano (incluyendo las operaciones propias de la estructura de espacio vectorial, el producto escalar y el producto vectorial). Relacionar estos conceptos algebraicos con ciertos elementos geométricos, como el ángulo formado por dos vectores.

- Conocer e identificar la expresión coordenada de los puntos del plano, los elementos notables de las rectas en el plano y sus diferentes expresiones en términos de ecuaciones.

- Conocer e identificar las posiciones relativas de un punto y una recta en el plano y en el espacio de manera gráfica y a través de sus expresiones algebraicas.

- Conocer e identificar las posiciones relativas de diferentes rectas en el plano y en el espacio de manera gráfica y a través de sus expresiones algebraicas.

A lo largo de la intervención pretendemos verificar la siguiente hipótesis (que llamaremos hipótesis nula): el modelo metodológico basado en la integración de Flipped Classroom con Aprendizaje Basado en Proyectos permite un incremento significativo del nivel de conocimientos sobre geometría analítica de vectores y rectas en el plano y el espacio en alumnado de $4 .^{\circ}$ ESO. Para la verificación de la hipótesis consideraremos dos variables cuantitativas, el pretest y el postest, que serán medidas utilizando los instrumentos que se describen a continuación.

\subsubsection{Instrumentos}

El instrumento específico utilizado para la verificación de la hipótesis nula ha sido un mismo cuestionario generalizado, pasado como pretest y postest, para todos los alumnos, realizado mediante la herramienta Kahoot, que consta de 13 preguntas de dificultad creciente (Anexo I). Todas las preguntas están cronometradas teniendo en cuenta el nivel de desarrollo de las mismas y el nivel curricular de los alumnos. Los resultados obtenidos en estos cuestionarios no han influido en la calificación de los alumnos en esta parte de la asignatura, que se midió en una prueba de evaluación posterior. 
METODOLOGÍA MIXTA FLIPPED CLASSROOM Y APRENDIZAJE BASADO EN PROYECTOS PARA EL APRENDIZAJE DE LA GEOMETRÍA ANALÍTICA EN SECUNDARIA

\subsubsection{Recursos y materiales}

Para el desarrollo de la intervención es necesario utilizar papel, útiles de escritura, pizarra (tradicional o digital), ordenador con conexión a internet y proyector. Los recursos digitales concretos que se han utilizado son los siguientes:

- Herramienta de gestión y banco de contenidos: software Google Classroom.

- Herramientas para edición de contenidos: software de presentaciones Prezi y murales tradicionales.

- Herramientas para controlar el proceso de aprendizaje: correo electrónico y observación directa sistemática.

- Herramienta de comunicación: software Google Classroom y correo electrónico.

\subsubsection{Desarrollo de la intervención}

La intervención se ha llevado a cabo en el segundo trimestre del curso 2019-20 a lo largo de doce sesiones (dos semanas y media), en las que se han realizado las siguientes actividades (Sánchez, 2020):

a. Sesión 1. Pretest y actividades de introducción-motivación. Tras el cuestionario de pretest, se proyectó un fragmento de un vídeo basado en el cuento Flatland, de Edwin Abbot (https://youtu.be/OgiO32MDq3k), relativo a la dimensionalidad geométrica, que dio origen a un debate acerca de los objetos geométricos que habitan en los espacios geométricos de diferentes dimensiones y acerca de la existencia, o no, de más de dos o tres dimensiones. Este debate sirvió a los propósitos de generar motivación en el alumnado, analizar sus conocimientos previos y definir de manera definitiva los diferentes proyectos a realizar.

b. Sesiones 2 a 11. Actividades de desarrollo de proyectos y explicación de contenidos. Los alumnos de cada una de las clases, distribuidos en ocho grupos diferentes, llevaron a cabo los diferentes proyectos que a continuación se exponen, propuestos por el profesor, pero cuyo alcance preciso, así como las estrategias de resolución y elaboración, cursan con la participación de los alumnos del correspondiente grupo. Adicionalmente, en tiempos intercalados con los momentos de trabajo autónomo en los proyectos, los grupos debieron presentar al resto de alumnos, a través de materiales diseñados por ellos mismos, ciertos contenidos geométricos. Además, cada grupo elaboró y llevó a cabo un diario reflexivo de la evolución de su trabajo a modo de seguimiento.

c. Sesión 12. Postest y evaluación. Los alumnos respondieron al cuestionario de postest a través de la herramienta Kahoot. Adicionalmente, realizaron una prueba de evaluación de contenido tipo examen. 
ÁLVARO ANTÓN SANCHO Y MARINA SÁNCHEZ DOMÍNGUEZ

METODOLOGÍA MIXTA FLIPPED CLASSROOM Y APRENDIZAJE BASADO EN PROYECTOS PARA EL APRENDIZAJE DE LA GEOMETRÍA ANALÍTICA EN SECUNDARIA

Describimos brevemente a continuación los diferentes proyectos que realizaron, por grupos, los alumnos de las dos clases (las llamaremos, por claridad expositiva, clase primera y clase segunda) en que se implementó la intervención, y las exposiciones orales que prepararon y desarrollaron a lo largo de las sesiones 2 a 11:

- Proyecto 1 (grupos 1 y 2). Maqueta sobre la posición relativa de dos rectas en el plano. Los alumnos de ambos grupos en cada una de las dos clases realizaron una maqueta tradicional, tipo mural en cartulina, ilustrando las diferentes posiciones relativas de las rectas en el plano. Además, cada grupo preparó y llevó a cabo una exposición teórica sobre los siguientes contenidos:

Grupo 1. Exposición sobre la estructura de espacio vectorial en el plano (suma de dos vectores y producto de un vector por un escalar).

Grupo 2. Exposición sobre la dependencia lineal de vectores en el plano y cálculo de las coordenadas del punto medio de un segmento dados los extremos.

- Proyecto 2 (grupo 3). Aplicaciones en la vida cotidiana de la posición relativa de dos rectas en el plano. Se trata de buscar y explicitar relaciones con el mundo real cotidiano de los contenidos geométricos propios del estudio de posiciones relativas de rectas en el plano. Para este proyecto, la metodología empleada por el grupo ha sido diversa, puesto que han realizado murales tradicionales (clase primera) y presentaciones mediante Prezi (clase segunda). Asimismo, este grupo preparó y llevó a cabo una exposición sobre el producto escalar de vectores en el plano y su aplicación al cálculo de la amplitud del ángulo formado por dos vectores.

- Proyecto 3 (grupo 4). Maqueta y aplicaciones en la vida cotidiana de la posición relativa de dos rectas en el espacio. De manera análoga al proyecto anterior, aquí se trata de estudiar las posiciones relativas de rectas en el espacio y conectar estos contenidos con el mundo cotidiano. Para este proyecto, la metodología empleada por el grupo ha sido el mural tradicional (clase primera) y la gamificación (clase segunda). En este último caso, los alumnos diseñaron un juego de pregunta-respuesta basado en buscar las diferentes posiciones de dos rectas en tres dimensiones en contextos de la vida real. Cabe destacar que, además, ilustraron las posiciones relativas de dos rectas en tres dimensiones, poniendo énfasis en las diferencias con el caso del plano. Adicionalmente, los alumnos del grupo (en ambas clases) prepararon una exposición sobre los elementos notables de una recta y sus diferentes expresiones en términos de ecuaciones.

- Proyecto 4 (grupos 5 y 6). Estudio de la posición relativa de dos rectas en el espacio a partir del conocimiento de la posición relativa de dos rectas en el plano. Los alumnos realizaron un estudio de las posiciones relativas de las rectas en el espacio como generalización del caso análogo en el plano e investigaron, en particular, diferentes casuísticas que se presentan al respecto. Para este proyecto, los alumnos diseñaron presentaciones Prezi 
ÁLVARO ANTÓN SANCHO Y MARINA SÁNCHEZ DOMÍNGUEZ

METODOLOGÍA MIXTA FLIPPED CLASSROOM Y APRENDIZAJE BASADO EN PROYECTOS

PARA EL APRENDIZAJE DE LA GEOMETRÍA ANALÍTICA EN SECUNDARIA

y murales tradicionales, según el grupo y la clase, a través de los cuales ilustraron las posiciones relativas de dos rectas en tres dimensiones, poniendo énfasis en las diferencias con el caso del plano. Cada grupo realizó, además, una exposición sobre los siguientes contenidos:

Grupo 5. Exposición sobre la posición relativa de un punto y una recta en el plano y sobre los conceptos de rectas coincidentes y paralelas.

Grupo 6. Exposición sobre los conceptos de rectas secantes y perpendiculares.

- Proyecto 5 (grupo 7). Distancias entre rectas en el plano. Se trata de analizar la distancia entre dos rectas en el plano según la posición relativa entre ellas. Para este proyecto, la metodología escogida por los alumnos del correspondiente grupo de ambas clases fue la tradicional, mediante el uso de pizarra y tiza. Asimismo, la exposición que llevaron a cabo ante sus compañeros abordaba la totalidad de los contenidos que abordaba su propio proyecto.

- Proyecto 6 (grupo 8). Dimensiones (idea del punto en el infinito). Los contenidos de este proyecto fueron particularmente introducidos en la primera sesión mediante un fragmento de vídeo explicativo extraído de la película Flatland y posterior debate sobre la existencia de la cuarta dimensión. El propósito principal de este trabajo fue que los alumnos investigaran el concepto de dimensión desde la perspectiva de la geometría analítica y concluyeran la existencia de la cuarta dimensión, que debería conducirles a la idea del punto en el infinito. Todo ello fue realizado por los alumnos mediante un documento de trabajo (clase primera) o una presentación Prezi (clase segunda), en los que, además, se introdujeron ciertas ideas intuitivas de la teoría de cuerdas. La exposición llevada a cabo por el grupo fue tocante a los contenidos geométricos trabajados en el proyecto.

\subsection{Análisis de resultados}

Consideramos, como hemos explicado, las variables pretest y postest, ambas cuantitativas y valoradas sobre la muestra de 50 alumnos de nuestro estudio. Vamos a estudiar estos datos ayudados por la herramienta SPSS de análisis estadístico. En Sánchez (2020) puede encontrarse un desarrollo más detallado de los datos que reportamos ahora. 

PARA EL APRENDIZAJE DE LA GEOMETRÍA ANALÍTICA EN SECUNDARIA

GRÁFICO 1

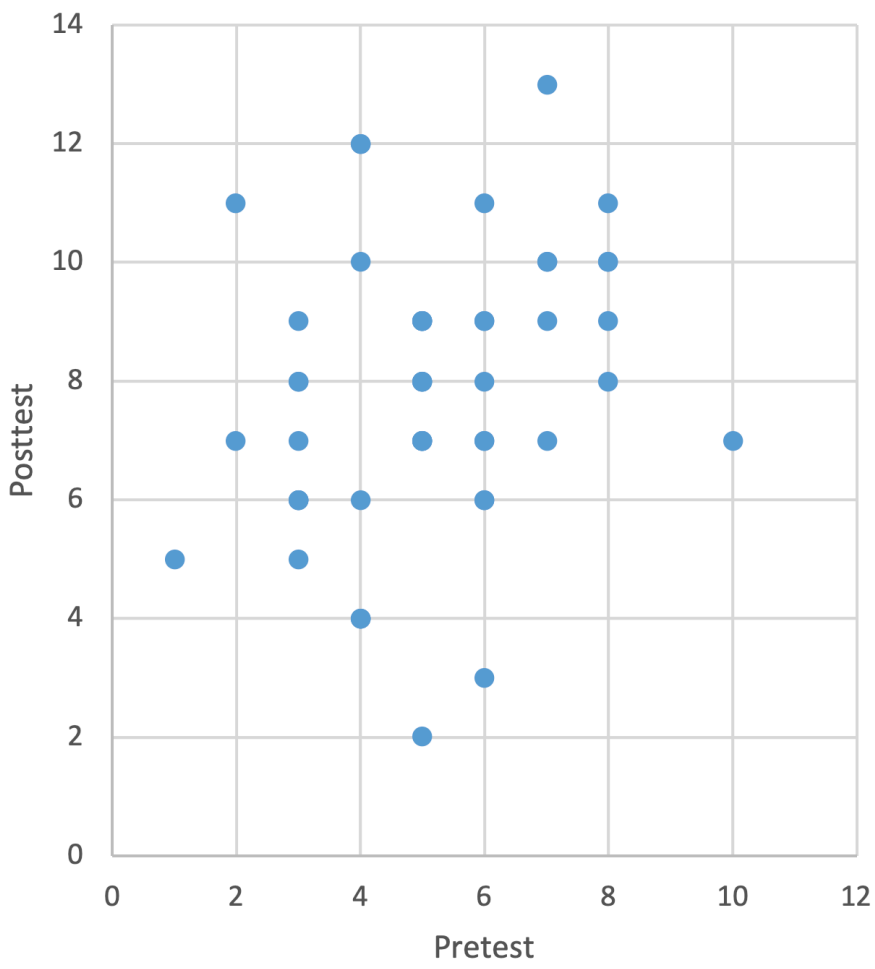

TABLA 2

Centralización y dispersión de las variables pretest y postest

\begin{tabular}{c|c|c}
\hline & Media & Desviación estándar \\
\hline Pretest & 5,12000 & 1,86963 \\
\hline Postest & 7,80000 & 2,35606 \\
\hline
\end{tabular}

Las medias indicadas en la Tabla 2 señalan que la mayoría de los alumnos han incrementado sus conocimientos con el modelo metodológico empleado. Además, las desviaciones típicas muestran que esas medidas medias son significativas. Esto sugiere, a priori, que el proceso de aprendizaje ha podido ser eficiente, aunque no haya, como parece derivarse del Gráfico 1, una correlación lineal entre las variables pretest y postest. A continuación, vamos a verificar todo esto aplicando un test de contraste de hipótesis. Cabe destacar, en todo caso, que los alumnos que han disminuido puntuación son exactamente aquellos que presentan necesidades 
especiales de aprendizaje y un alumno repetidor. Interpretamos que la razón de este hecho es que no se ha hecho adaptación alguna en los cuestionarios de pretest y postest para contrarrestar la dificultad que estos alumnos pueden encontrarse cuando se enfrentan a una prueba de ese nivel y en las circunstancias temporales y cronométricas en que se realizó.

Aplicamos la prueba de Lilliefors con nivel de significación del $95 \%$ para analizar el grado de ajuste a la distribución normal de las variables pretest y postest (Tabla 3). Como el p-valor es mayor a 0,05 en el caso del postest y ligeramente menor, pero muy próximo, a 0,05 en el caso del pretest, podemos asumir que ambas variables siguen una distribución normal.

TABLA 3

Prueba de normalidad de Lilliefors

\begin{tabular}{c|c|c}
\hline Estadístico & Gl & Sig. \\
\hline 0,126 & 50 & 0,047 \\
\hline 0,107 & 50 & 0,200 \\
\hline
\end{tabular}

En la Tabla 4 mostramos los resultados de la aplicación de un test de contraste para muestras apareadas, con el fin de comprobar si hay una correlación lineal entre las variables pretest y postest.

TABLA 4

Contraste de correlación lineal para muestras apareadas

\begin{tabular}{c|c|c|c|c}
\hline & Media & $N$ & $\begin{array}{c}\text { Desviación } \\
\text { estándar }\end{array}$ & $\begin{array}{c}\text { Media de error } \\
\text { estándar }\end{array}$ \\
\hline Pretest & 5,1200 & 50 & 1,86963 & 0,26441 \\
\hline Postest & 7,8000 & 50 & 2,35606 & 0,33320 \\
\hline $\begin{array}{c}\text { Correlaciones de } \\
\text { muestras emparejadas }\end{array}$ & & & & \\
\hline & $N$ & Correlación & Sig & \\
\hline & $\begin{array}{c}\text { Par Pretest- } \\
\text { Postest }\end{array}$ & 50 & 0,279 & 0,050 \\
\hline
\end{tabular}

Observamos que la correlación entre las variables pretest y postest, 0,279, manifiesta una relación lineal débil entre ambas. Además, como el p-valor es 0,05, podemos asumir que no existe correlación lineal entre el pretest y el postest. Sin embargo, si aplicamos la prueba T de muestras emparejadas, obtenemos los resultados que se indican en la Tabla 5. 
ÁLVARO ANTÓN SANCHO Y MARINA SÁNCHEZ DOMÍNGUEZ

METODOLOGÍA MIXTA FLIPPED CLASSROOM Y APRENDIZAJE BASADO EN PROYECTOS PARA EL APRENDIZAJE DE LA GEOMETRÍA ANALÍTICA EN SECUNDARIA

TABLA 5

Prueba T de muestras emparejadas

\begin{tabular}{|c|c|c|c|c|c|c|c|c|}
\hline & \multirow[t]{2}{*}{ Media } & \multirow{2}{*}{$\begin{array}{l}\text { Desv. } \\
\text { estándar }\end{array}$} & \multirow{2}{*}{$\begin{array}{c}\text { Media } \\
\text { error } \\
\text { estándar }\end{array}$} & \multicolumn{2}{|c|}{$\begin{array}{l}95 \% \text { confianza } \\
\text { de la diferencia }\end{array}$} & \multirow[t]{2}{*}{$T$} & \multirow[t]{2}{*}{$G l$} & \multirow[t]{2}{*}{$\begin{array}{c}\text { Sig. } \\
\text { (bilat.) }\end{array}$} \\
\hline & & & & Inf. & sup. & & & \\
\hline $\begin{array}{l}\text { Par Pretest- } \\
\text { Postest }\end{array}$ & 2,680 & 2,567 & 0,363 & $-3,410$ & $-1,950$ & $-7,382$ & 49 & 0,000 \\
\hline
\end{tabular}

Siendo el p-valor igual a 0 , menor que el nivel de significación, 0,05, podemos rechazar la hipótesis nula y aceptar, en consecuencia, que existen diferencias significativas entre los niveles inicial y final de conocimiento de los alumnos. Siendo así que las medias de los resultados de los cuestionarios inicial y final muestran un incremento (también significativo, según muestran las desviaciones típicas), podemos considerar verificada nuestra hipótesis de partida, esto es, que la metodología implementada suscita un incremento significativo en el nivel de conocimientos sobre geometría analítica vectorial de los alumnos de $4 .^{\circ}$ de ESO.

\section{CONCLUSIONES}

Nuestra intervención educativa ha mostrado, sin atisbo de duda, que la metodología objeto de análisis ha sido eficiente a la hora de suscitar el aprendizaje de los conceptos de la geometría analítica en un nivel de $4 .^{\circ}$ de ESO. A nuestro entender, este hecho se deriva de que la inherente conexión entre el trabajo por proyectos y el FC ha conseguido aunar los beneficios didácticos que la literatura señala para cada modelo por separado.

La motivación del alumnado hacia el aprendizaje de los contenidos trabajados es, asimismo, digna de ser destacada. El índice de aceptación de la propuesta didáctica fue inicialmente muy alto, aunque surgieron inquietudes en los alumnos a medida que se desgranaba el método. Estas dudas concernían, principalmente, a su autoconcepto y giraban en torno a su falta de confianza en su capacidad para aprender a aprender y para conseguir enseñar a sus compañeros contenidos que ellos mismos desconocían al principio. Sin embargo, esas inquietudes fueron desapareciendo según avanzaba la dinámica del aula, fue creciendo la autoconfianza y, con ello, la motivación. Las exposiciones fueron, en general, satisfactorias y originales, aunque con variaciones en la profundidad del tratamiento de los contenidos.

Hemos apreciado, asimismo, diferencias actitudinales entre el alumnado de itinerario "científico-técnico» respecto de aquel orientado a «ciencias sociales». Los primeros eran, en general, más participativos y preparaban más concienzudamente el contenido, probablemente debido a un interés previo más elevado hacia la materia, fruto del itinerario académico escogido. 
Este estudio está limitado, tanto en el tamaño de la muestra escogida como, sobre todo, en el nivel en que se ha llevado a cabo y los contenidos específicos trabajados. Proponemos, como líneas de estudio futuras, emplear esta metodología mixta en alumnado de otros niveles, incluso adaptarla a otras etapas educativas, y explorar la manera de aplicarla a contenidos curriculares diversos dentro del área de matemáticas. Valdría la pena, asimismo, validar esta metodología desde el punto de vista de una investigación con grupo experimental y grupo control, algo que nosotros no hemos podido llevar a cabo por razones de organización de centro.

\section{REFERENCIAS BIBLIOGRÁFICAS}

Aguilera-Ruiz, C., Manzano-León, A., Martínez-Moreno, I., Lozano-Segura, M. C. y Casiano Yanicelli, C. (2017). El modelo Flipped Classroom. International Journal of Developmental and Educational Psychology, 4 (1), 261-266.

Badia, A. y García, C. (2006). Incorporación de las TIC en la enseñanza y el aprendizaje basados en la elaboración colaborativa de proyectos. Revista de Universidad y Sociedad del Conocimiento, 2 (3), 42-54.

Barron, B. J. S., Schwartz, D. L., Vye, N. J., Moore, A., Petrosino, A., Zech, L., Bransford, J. D. y The Cognition and Technology Group at Vanderbilt. (1998). Doing with understanding: Lessons from research on problem- and project-based learning. The Journal of the Learning Sciences, 7, 271-311.

Boaler, J. (1998). Open and closed mathematics: Student experiences and understandings. Journal for Research in Mathematics Education, 29, 41-62.

Boaler, J. (2002). Experiencing School Mathematics: Traditional and Reform Approaches to Teaching and their Impacto on Student Learning. New Jersey: Lawrence Erlbaum Associates.

Cronhjort, M., Filipsson, L. y Weurlander, M. (2018). Improved engagement and learning in flipped-classroom calculus. Teaching Mathematics and its Applications, 37 (3), 113-121.

Dole, S., Bloom, L. y Kowalske, K. (2016). Transforming Pedagogy: Changing Perspectives from TeacherCentered to Learner-Centered. Interdisciplinary Journal of Problem-Based Learning, 10 (1). Descargado el día 13 de mayo de 2019. https://doi.org/10.7771/15415015.1538.

Flumerfelt, S. y Green, G. (2013). Using Lean in the Flipped Classroom for At Risk Students. Educational Technology \& Society, 16 (1), 356-366.

Fornons Jou, V. y Palau Martín, R. F. (2016). Flipped Classroom en la asignatura de matemáticas de $3 .^{\circ}$ de Educación Secundaria Obligatoria. Edutec. Revista Electrónica de Tecnología Educativa, (55), a322. Descargado el día 13 de mayo de 2019. https://doi. org/10.21556/edutec.2016.55.284

García, A. (2016). Aprendizaje inverso y motivación en el aula universitaria. Revista Pulso, 39, 199-218.

Gómez-Chacón, I. (2000). Matemática emocional. Los afectos en el aprendizaje matemático. Madrid, España: Narcea.

Hamdan, N., McKnight, P., McKnight, K. y Arfstrom, K. M. (2013). Review of Flipped Learning. Flipped Learning Network. Pearson. George Mason University 10.4236/ce. Descargado el día 13 de mayo de 2019. https://flippedlearning.org/wp-content/uploads/2016/07/ LitReview_FlippedLearning.pdf 
Hutchings, M. y Quinney, A. (2015). The Flipped Classroom Disruptive Pedagogies Enabling Technologies and Wicked Problems Responding to the Bomb in the Basement. Electronic Journal of e-Learning, 13 (2), 106-119.

James, A., Chin, C. y Williams, B. (2014). Using the flipped classroom to improve student engagement and to prepare graduates to meet maritime industry requirements: a focuson maritime education. WMU Journal of Maritime Affairs, 13 (2), 331-343.

Lage, M., Platt, G. y Treglia, M. (2000). Inverting the classroom: A gateway to creating an inclusive learning environment. The Journal of Economic Education, 31 (1), 30-43.

Leicht, R. M., Zappe, S. E., Messner, J. I., Litzinger, T. y State, P. (2012). Employing the classroom flip to move «lecture» out of the classroom. Journal of Applications and Practices in Engineering Education, 3 (1), 19-31.

Martín, R. D. y Calvillo, A. (Coords.). (2017). The flipped learning: una guí gamificada para novatos y no tan novatos. Madrid: Ediciones UNIR.

Mettas, A. C. y Constantinou, C. C. (2007). The technology fair: a project based learning approach for enhancing problema solving skills and interest in design and technology education. International Journal of Technology and Design Education, 18, 79-100.

Morales Maure, L. y García Marimón, O. (2013). La afectividad de la inteligencia. Formación Universitaria, 6 (5), 3-12.

Morales Maure, L. y García Marimón, O. (2015). Un aprendizaje basado en proyecto en matemática con alumnos de undécimo grado. NÚMEROS. Revista de Didáctica de las Matemáticas, 90, 21-30.

OCDE. (2020). A note about Spain in PISA 2018: Further analysis of Spain's data by testing date (updated on 23 July 2020). Descargado el 28 de noviembre de 2020. https://www. oecd.org/pisa/PISA2018-AnnexA9-Spain.pdf

Phillips, C. y Trainor, J. (2014). Millennial students and the flipped classroom. Proceedings of the American Society of Business and Behavioral Sciences, 21 (1), 519-530.

Pujolàs, P. (2004). Aprender juntos alumnos diferentes: Los equipos de aprendizaje cooperativo en el aula. Barcelona: Octaedro.

Real Decreto 1105/2014, de 26 de diciembre, por el que se establece el currículo básico de la Educación Secundaria Obligatoria y del Bachillerato.

Rigo, D. Y., Riccetti, A. E., Siracusa, M. y Paoloni, P. (2019). Tres experiencias sobre clases invertidas para promover el compromiso por el aprendizaje. Percepciones de estudiantes universitarios. Páginas de Educación, 12 (2), 43-58.

Roehl, A., Reddy, S. y Shannon, G. (2013). The flipped classroom: an opportunity to engage millennial students through active learning strategies. Journal of Family and Consumer Sciences, 105 (2), 44-49.

Sánchez Domínguez, M. (2020). Flipped Classroom y aprendizaje Basado en Problemas aplicados a la enseñanza secundaria de las matemáticas. Trabajo Fin de Máster. Ávila: Universidad Católica de Ávila.

Santiago, R. y Tourón, J. (2015). Claves para entender el modelo de aprendizaje inverso: una aproximación al Flipped Learning. Harvard Deusto Learning \& Pedagogics, 2, 32-39.

Thomas, J. W. (2000). A review of research on project-based learning. Descargado el 13 de mayo de 2019. http://www.bobpearlman.org/BestPractices/PBL_Research.pdf.

Vaello Orts, J. (2009). El profesor emocionalmente competente. Barcelona: Graó.

Wilson, S. G. (2013). The Flipped Class: A Method to Address the Challenges of an Undergraduate Statistics Course. Teaching of Psychology, 40 (3), 193-199. 
Anexo I. Cuestionario de Pretest-postest. Extraído de SÁnChez (2020)

1. La dimensión de un punto es 1.
A) Verdadero
B) Falso

2. La dimensión de una recta es 1.
A) Verdadero
B) Falso

3. Las coordenadas del vector de origen y de extremo son:
A)
B)
C)

4. ¿Son los siguientes vectores equipolentes?

\section{FIGURA 1}

Vectores
A) Sí
B) $\mathrm{No}$
C) Solo si lo desplazamos

5. Sean y dos puntos del plano. El punto medio del segmento es:
A)
B)
C)
D)

6. Los vectores y son perpendiculares.
A) Verdadero
B) Falso

7. La pendiente de la recta es
A)
B)
C)
D)

8. La posición relativa de estas rectas es 

PARA EL APRENDIZAJE DE LA GEOMETRÍA ANALÍTICA EN SECUNDARIA

\section{FIGURA 2}

\section{Rectas}
A) Coincidentes
B) Secantes
C) Paralelas
D) Perpendiculares

9. Las rectas perpendiculares son un caso particular de rectas secantes
A) Verdadero
B) Falso

10. La posición relativa de las rectas, es
A) Coincidentes
B) Secantes
C) Paralelas
D) Perpendiculares

11. La ecuación general de la recta que pasa por los puntos y es:
A)
B)
C)
D)

12. La ecuación de una recta paralela a es
A)
B)
C)

13. La ecuación de la recta perpendicular a y que pasa por el punto es
A)
B)
C)
D) 